\title{
Atratividade de adultos de Chrysoperla externa (Hagen, 1861) aos compostos voláteis de coentro, endro e erva-doce (Apiaceae) em condições de laboratório ${ }^{1}$
}

\author{
André Luis Santos Resende ${ }^{* 2}$, Roberta Botelho Ferreira ${ }^{3}$, Brígida Souza ${ }^{4}$ \\ http://dx.doi.org/10.1590/0034-737X201562010005
}

\section{RESUMO}

Espécies de Apiaceae dispõem de óleos essenciais, nos quais podem ocorrer compostos voláteis, que funcionam como sinais para atração e manutenção de inimigos naturais nas áreas cultivadas. Com base nestas características, este trabalho objetivou avaliar a atratividade aos adultos do predador Chrysoperla externa. Foram utilizados folhas e caules de coentro, endro e erva-doce, coletados aos 30 e 60 dias após a semeadura. As plantas foram dispostas em olfatômetro de quatro vias (formato de "X") disponibilizando-se os odores para machos e fêmeas, virgens e acasalados, em testes de livre escolha. Ao serem liberados individualmente no interior do olfatômetro, foram cronometrados cinco minutos e contabilizado o tempo total de permanência do inseto em cada braço do aparelho. Os dados foram analisados pelo teste $\chi^{2}$, com frequência esperada de $25 \%$. Estudou-se o rendimento de óleo essencial das três espécies de plantas, 30 e 60 dias após a semeadura, utilizando-se do método de hidrodestilação. A composição química dos óleos foi determinada por cromatografia gasosa acoplada a espectrômetro de massas. Verificou-se que adultos virgens têm preferência por plantas de coentro, enquanto os acasalados preferem plantas de erva-doce, ambas coletadas aos 30 dias. Plantas com 60 dias não proporcionaram resposta atrativa aos adultos de C. externa. O rendimento de óleo tendeu a aumentar com o desenvolvimento fenológico da planta. A composição química do óleo de coentro revelou, como componentes majoritários, o (2E)-decenal e decanal e, para erva-doce, a maior concentração foi de (E)-anetol.

Palavras-chave: Crisopídeos, óleo essencial, Coriandrum sativum L., Anethum graveolens L., Foeniculum vulgare Mill.

\section{ABSTRACT}

\section{Attractiveness of Chrysoperla externa (Hagen, 1861) adults to volatile compounds of coriander, dill and fennel (Apiaceae) in laboratory conditions}

Species of Apiaceae have essential oils that may present volatile compounds that act as signals to attract and maintain natural enemies in the crop. Based on these characteristics, the objective of this study was to evaluate the attractiveness to adults of the predator Chrysoperla externa. Leaves and stems of plants of coriander, dill and fennel, collected at 30 and 60 days after sowing, were used here. The plants were arranged in four-way olfactometer ("X" format), providing odor to males and females, virgin and mated insects, in free choice tests. When released individually inside the olfactometer, five minutes were timed and the total time spent by the insect in each arm of the device was counted. Data were analyzed by the $\div 2$ test, with expected frequencies of $25 \%$. The yield of the

\footnotetext{
Submetido em 23/11/2012 e aprovado em 24/01/2014

'Este trabalho é parte da tese de doutorado do primeiro autor.

${ }^{2}$ Universidade Federal de Lavras, Departamento de Entomologia, Lavras, Minas Gerais, Brasil. alsresende@yahoo.com.br

${ }^{3}$ Universidade Federal de Lavras, Departamento de Entomologia, Lavras, Minas Gerais, Brasil. robertabotelhoferreira@yahoo.com.br

${ }^{4}$ Universidade Federal de Lavras, Departamento de Entomologia, Lavras, Minas Gerais, Brasil. brgsouza@ den.ufla.br

*Autor para correspondência: alsresende@yahoo.com.br
} 
essential oil of three species of plants at 30 and 60 days after sowing was evaluated by using the method of hydrodistillation. The chemical composition of the oils was determined by gas chromatography coupled with mass spectrometer. It was found that virgin adults have a preference for coriander plants, while the mated insects prefer fennel, both collected at 30 days. Plants at 60 days did not provide attractiveness response to adults of C. externa. The oil yield tended to increase with the phenological development of the plant. The chemical composition of coriander oil revealed (2E)-decenal and decanal as major components, and the highest concentration was of (E)anethole for fennel.

Key words: Anethum graveolens L., Coriandrum sativum L., essential oil, Foeniculum vulgare Mill, Lacewings.

\section{INTRODUÇÃO}

Entre as inúmeras espécies de plantas aromáticas, aquelas da família Apiaceae veem sendo investigadas para utilização como plantas companheiras, pela atração que exercem sobre os inimigos naturais de insetos-pragas (Lixa et al., 2010; Resende et al., 2010). Essas espécies são caracterizadas por liberarem uma quantidade relativamente grande de compostos voláteis, presentes nos óleos essenciais que produzem (Simões \& Spitzer, 2000).

Os óleos essenciais são substâncias complexas, com fragrância variável, e estão presentes, além de nas apiáceas, em plantas de Asteraceae, Lamiaceae, Lauraceae, Myrtaceae, Myristicaceae, Piperaceae e Rutaceae (Simões \& Spitzer, 2000). Os compostos voláteis, geralmente, são oriundos de tecidos vegetativos e fazem parte do sistema de defesa da planta. Esses compostos atuam de forma a repelir micro-organismos e animais, ou a atrair inimigos naturais destes, protegendo-as indiretamente por meio de interações tritróficas. Com essa estratégia, a própria planta pode reduzir o número de herbívoros (Kessler \& Baldwin, 2001). Entre os inimigos naturais comumente associados a plantas cultivadas encontram-se os crisopídeos (Neuroptera: Chrysopidae), cujas larvas são predadores que exercem importante papel no controle de diversos artrópodes-praga, presentes em vários agroecossistemas. Cochonilhas, pulgões, moscas-brancas, tripes, pequenas larvas de besouros e de lepidópteros, ácaros, entre outros, constituem-se em presas desses inimigos naturais (Carvalho \& Souza, 2002; Freitas, 2002; Costa et al., 2003).

Adultos de crisopídeos, os quais são glicopolinívoros, apresentam hábito crepuscular ou noturno e, por isso, o olfato é o principal sentido utilizado no forrageamento, já que a visão é prejudicada pela baixa intensidade luminosa. A atratividade de adultos de Chrysoperla carnea (Stephens, 1836) a voláteis foi estudada a partir de compostos sintéticos presentes em repolho (Reddy et al., 2002). A atratividade dessa espécie e de Chrysopa oculata Say, 1839 também foi testada aos voláteis presentes em folhas e flores de alfafa, verificando-se que (Z)-3- hexenil-acetato e 2-feniletanol foram atrativos a ambas (Zhu et al., 2005). Estudos dessa natureza estão restritos a espécies de ocorrência holártica, sendo evidente a carência de conhecimento relacionado com aquelas de distribuição neotropical, como Chrysoperla externa (Hagen, 1861), por exemplo, uma das espécies mais comuns e mais estudadas nessa região.

Assim, o objetivo deste trabalho foi avaliar a atratividade de voláteis emitidos por três espécies de plantas de Apiaceae a adultos de C. externa, em laboratório.

\section{MATERIAL E MÉTODOS}

O estudo foi conduzido com adultos de C. externa provenientes da criação do Departamento de Entomologia da Universidade Federal de Lavras (UFLA), Lavras, MG. Foram utilizadas plantas de coentro (Coriandrum sativum L.), endro (Anethum graveolens L.) e erva-doce (Foeniculum vulgare Mill.), cultivadas em casa de vegetação, em vasos de $10 \mathrm{~L}$ com terra e substrato de plantio (2:1). Aos 30 dias, procedeu-se à adubação de cobertura com sulfato de amônio, na dosagem equivalente a $50 \mathrm{Kg}$ $\mathrm{ha}^{-1}$. A aplicação de água foi feita a cada dois dias.

Utilizou-se um olfatômetro em X, de múltipla escolha, construído com vidro transparente, com braços de $15 \mathrm{~cm}$ de comprimento e $3 \mathrm{~cm}$ de diâmetro e com um tubo perpendicular de mesmo diâmetro, na parte ventral da interseção dos braços. Esse conjunto foi disposto sobre uma mesa com tampo de isopor, com um furo central de quatro centímetro de diâmetro, onde foi encaixado o braço perpendicular. Cada braço do $\mathrm{X}$ foi conectado a um recipiente com volume de 1,3 $\mathrm{L}$, os quais, por sua vez, foram conectados a uma bomba de aquário calibrada para um fluxo de ar de $1,5 \mathrm{~L} \mathrm{~min}^{-1}$ (Figura 1).

Um estudo prévio do deslocamento do ar dentro do olfatômetro, utilizando-se água e gelo seco, permitiu certificar não haver mistura do ar entre os diferentes braços. Foi, então, feita uma pequena marca com tinta na metade do comprimento de cada braço. Atribuiu-se a escolha dos insetos por um ou outro odor quando ultrapassavam completamente a marca. 
Em teste preliminar, foi estudada a atratividade da parte aérea (folhas e caule) de coentro, endro e erva-doce para adultos de C. externa. Esse teste consistiu na introdução de apenas uma espécie de planta por vez, no interior do olfatômetro, mantendo-se os outros três recipientes vazios. Para cada uma das aromáticas testadas procedeu-se à liberação individualizada de 30 espécimes recém-emergidos (15 machos e 15 fêmeas). O tempo total de permanência dos insetos na parte do olfatômetro correspondente às aromáticas foi de $43,41 \%$ para endro, $44,39 \%$ para erva-doce e $45,25 \%$ para coentro, todos significativos pelo teste $\chi^{2}(\mathrm{p} \leq 0,05)$ com frequência esperada de $25 \%$.

De posse desses resultados, efetuou-se o teste de livre escolha, utilizando-se simultaneamente as três espécies de plantas. Os tratamentos consistiram em buquês constituídos por folhas (com pecíolos) de coentro, endro e erva-doce, coletadas aleatoriamente e dispostas no interior dos recipientes do olfatômetro. As folhas tiveram o pecíolo inserido em um tubo de ensaio com água para evitar o murchamento. Um recipiente com apenas um tubo de ensaio com água foi utilizado como testemunha.

Os insetos foram colocados individualmente no interior de tubos de ensaio e, no momento da liberação, cada tubo era aproximado do braço perpendicular do olfatômetro. As cronometragens foram efetuadas durante cinco minutos, a partir do momento em que atingiam o centro do "X", contabilizando-se o tempo total de permanência em cada braço correspondente a cada tratamento.

Os buquês foram trocados a cada 25 insetos liberados e o vidro em formato de " $X$ " foi lavado com água e detergente e secado com secador de ar quente manual, a cada dez insetos estudados. Por ocasião da retirada do vidro para limpeza, trocava-se aleatoriamente a posição de encaixe de cada tratamento.

Os testes foram realizados com plantas colhidas aos 30 e 60 dias após a semeadura. Foram utilizados 50 machos e 50 fêmeas, sendo 25 exemplares virgens, recém-

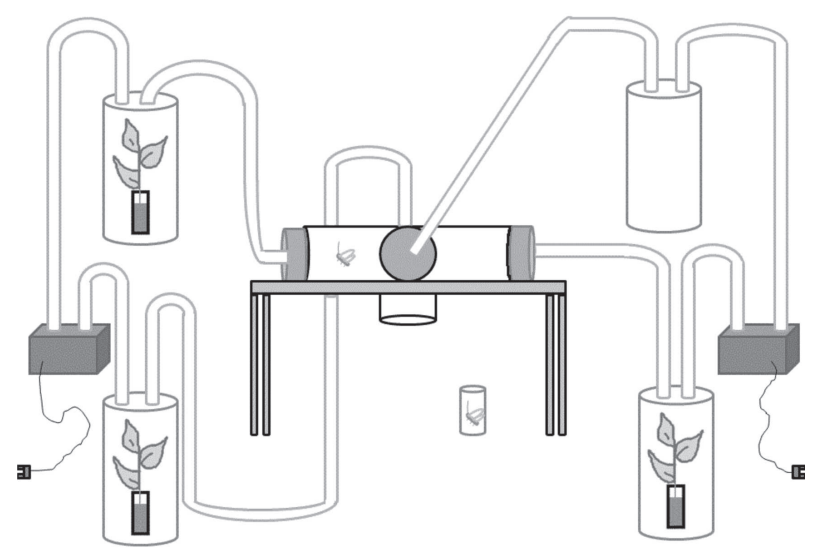

Figura 1. Desenho esquemático do olfatômetro de múltipla escolha em formato $X$. emergidos, e 25 supostamente acasalados, uma vez que foram mantidos juntos durante sete dias após a emergência. Nesse período, esses insetos foram alimentados com dieta composta de lêvedo de cerveja e mel, na proporção de 1:1.

Determinou-se o rendimento de óleos essenciais das três espécies de plantas, a partir de material fresco coletado em casa de vegetação, aos 30 e 60 dias após a emergência. A extração foi realizada no Laboratório de Química Orgânica do Departamento de Química da UFLA, utilizando-se o método de hidrodestilação (Clevenger modificado). Utilizaram-se três amostras de $30 \mathrm{~g}$ do material vegetal fresco para cada espécie vegetal para a extração do óleo essencial, hidrodestilando-se as amostras durante $2 \mathrm{~h}$. Ao final desse período, coletou-se o hidrolato, que foi fracionado com diclorometano. A fase orgânica, composta pelo óleo essencial + diclorometano, foi acrescida de sulfato de sódio anidro e, em seguida, filtrada. Após esse processo, as amostras foram colocadas num rota-evaporador para evaporação do solvente.

A composição química dos óleos essenciais foi determinada a partir de amostras únicas, constituídas pelos óleos essenciais extraídos aos 30 e 60 dias, sendo realizadas apenas para o coentro e a erva-doce, já que apresentaram respostas significativas aos crisopídeos. A análise qualitativa do óleo essencial foi realizada por cromatografia em fase gasosa acoplada à espectrometria de massa (CG/EM - Shimadzu, modelo QP 5050A), na Universidade Federal de Sergipe, conforme metodologia descrita em Andrade et al. (2012), em que foram utilizadas as seguintes condições experimentais: coluna capilar de sílica fundida ( $30 \mathrm{~m}$ x 0,25 mm), com fase ligada DB5 (0,25 $\mu \mathrm{m}$ de espessura de filme); He como gás de arraste, com fluxo de $1,0 \mathrm{~mL} / \mathrm{min}$; temperatura programada, mantendo-se $50{ }^{\circ} \mathrm{C}$ por $1,5 \mathrm{~min}$, seguindo-se de aumento de $4{ }^{\circ} \mathrm{C} / \mathrm{min}$, até atingir $200{ }^{\circ} \mathrm{C}$, depois a 10 ${ }^{\circ} \mathrm{C}$, até atingir $250{ }^{\circ} \mathrm{C}$, mantendo-se constante essa temperatura por $5 \mathrm{~min}$; temperatura do injetor de $250{ }^{\circ} \mathrm{C}$ e temperatura do detector (ou interface) de $280{ }^{\circ} \mathrm{C}$; volume da amostra injetada de $0,5 \mu \mathrm{L}$ em acetato de etila; taxa de partição do volume injetado de 1:100 e pressão na coluna de $64,20 \mathrm{kPa}$. As condições do espectrômetro de massas foram: operando por impacto de elétrons (70 $\mathrm{eV}$ ), com detector de varredura 1.000; intervalo de varredura de 0,50 fragmento e fragmentos detectados na faixa de 40 a 500 Daltons.

A identificação dos constituintes foi realizada com base na comparação dos índices calculados para cada composto com índices de retenção da literatura (Adams, 2007). Para a determinação do índice de retenção, foi utilizada a equação de Van den Dool e Kratz (Van den Dool \& Kratz, 1963), em relação à série homóloga de 
n-alcanos $\left(\mathrm{nC}_{8}-\mathrm{nC}_{18}\right)$ e fazendo-se extrapolação para $\mathrm{C}_{19}$ $\mathrm{e}_{20}$. Também foram utilizadas duas bibliotecas do equipamento NIST107 e NIST21, que permitem a comparação dos dados dos espectros de massa obtidos com aqueles existentes nas bibliotecas. A análise quantitativa foi obtida pela integração do cromatograma total de íons (Rosado et al., 2011).

Os dados obtidos no teste do olfatômetro foram expressados em percentagem de tempo total e submetidos ao teste de qui-quadrado $\left(\chi^{2}\right)$ com frequência esperada de $25 \%$ e probabilidade de $5 \%$. Para o rendimento de óleo, foi considerado o delineamento inteiramente casualizado, sendo os dados comparados pelo teste de Fischer ( $\leq \leq 0,05)$, para a época (30 e 60 dias), e pelo teste de Tukey $(\mathrm{p} \leq 0,05)$, para as plantas (coentro, endro e erva-doce).

\section{RESULTADOS E DISCUSSÃO}

No teste com folhas colhidas aos 30 dias, os adultos virgens (machos e fêmeas) foram atraídos por coentro, enquanto os acasalados foram mais atraídos por erva-doce (Figura 2). Machos e fêmeas de Chrysoperla carnea também responderam semelhantemente quando submetidos a testes de livre escolha, utilizando-se plantas de berinjela, quiabo e pimenta infestadas por ácaros (Zhu et al., 2005). Testando vários compostos voláteis, Reddy et al. (2002) também constataram respostas semelhantes entre machos e fêmeas de C. carnea e afirmaram que machos e fêmeas de crisopídeos possivelmente apresentam a mesma resposta aos estímulos olfativos.
Assim como os crisopídeos, coccinelídeos (Coleoptera) também foram atraídos por plantas de coentro, endro e erva-doce, cultivadas em campo, as quais lhes serviram como sítio de acasalamento e oviposição (Lixa et al., 2010). A presença constante de joaninhas, em todas as fases de desenvolvimento, também foi constatada em cultivo de couve consorciada com coentro, mesmo não havendo colônias de pulgões na couve (Resende et al., 2010; 2011).

Além da atratividade a inimigos naturais, plantas de coentro podem promover a redução da infestação de alguns insetos fitófagos. Togni et al. (2010) verificaram que a inclusão de voláteis de coentro junto aos de tomate reduzem a atração de Bemisia tabaci (Gennadius, 1889), biótipo B, pela solanácea. Portanto, o uso de coentro em cultivo consorciado pode auxiliar na diminuição de insetos-praga pelo efeito direto sobre eles, por afetar a escolha do hospedeiro, ou indireto, pela manutenção de inimigos naturais (Medeiros et al., 2009, Togni et al., 2009).

No teste com folhas colhidas aos 60 dias, os adultos (machos e fêmeas) acasalados e as fêmeas virgens sinalizaram um comportamento de não preferência aos odores das apiáceas testadas, haja vista terem permanecido por mais tempo no eixo vazio do olfatômetro. Os machos virgens não apresentaram preferência por nenhum tratamento (Figura 3), uma vez que o tempo de permanência nos quatro eixos foi aleatório ( $\mathrm{p}>0,05)$.

Portanto, coentro, endro e erva-doce com 60 dias não são indicados para a manutenção de $C$. externa na área de cultivo, considerando-se a ação direta das plantas pela emissão de voláteis. No entanto, deve-se levar em conta

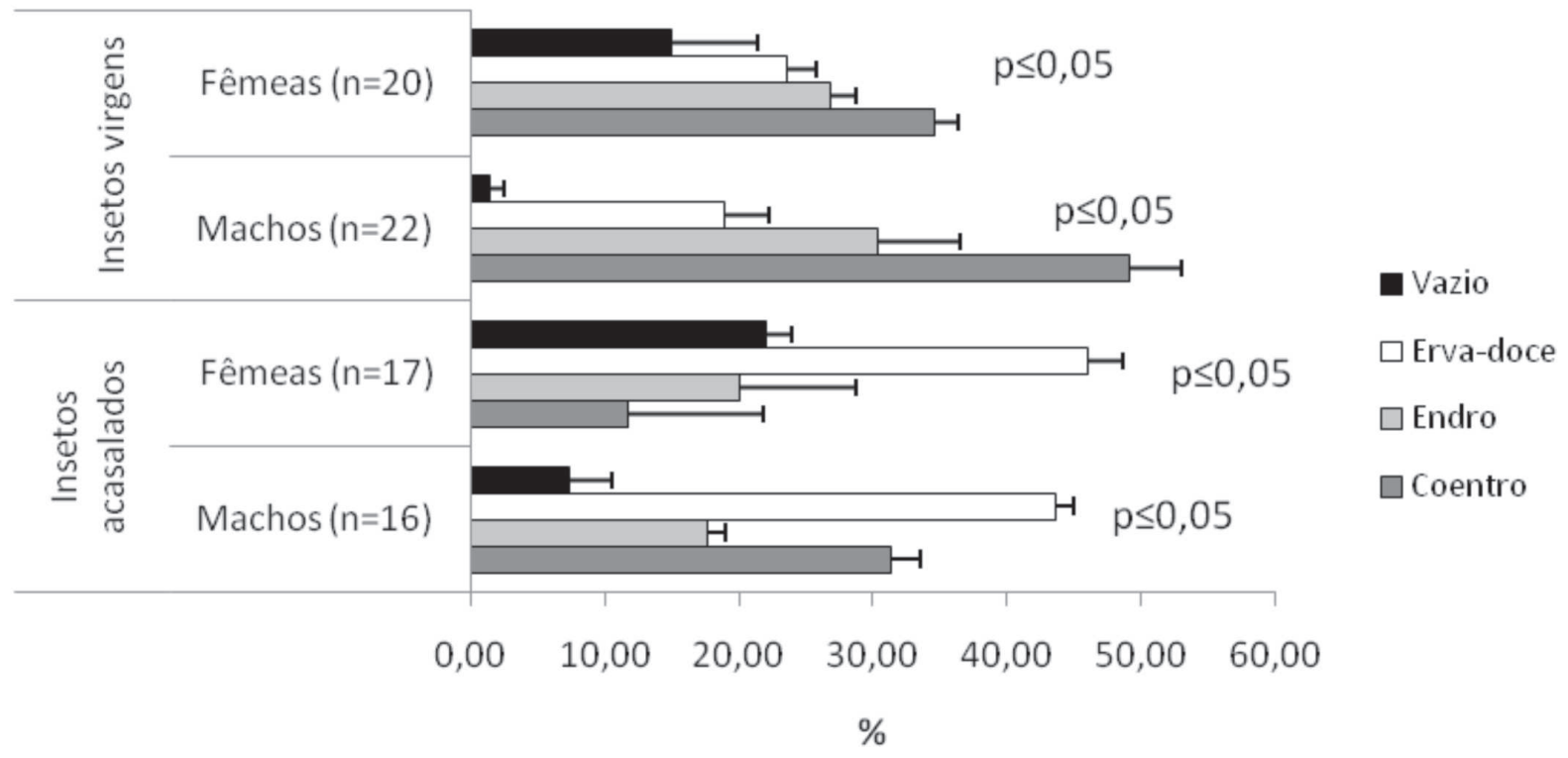

Figura 2. Percentagem de tempo total (médias \pm erro padrão) de machos e fêmeas de Chrysoperla externa, acasalados e virgens, em teste de múltipla escolha em olfatômetro contendo plantas de coentro, endro e erva-doce com 30 dias após a semeadura. ( $\mathrm{p} \leq 0,05$ representa percentagens não aleatórias pelo teste $\left.\chi^{2}\right)$. 


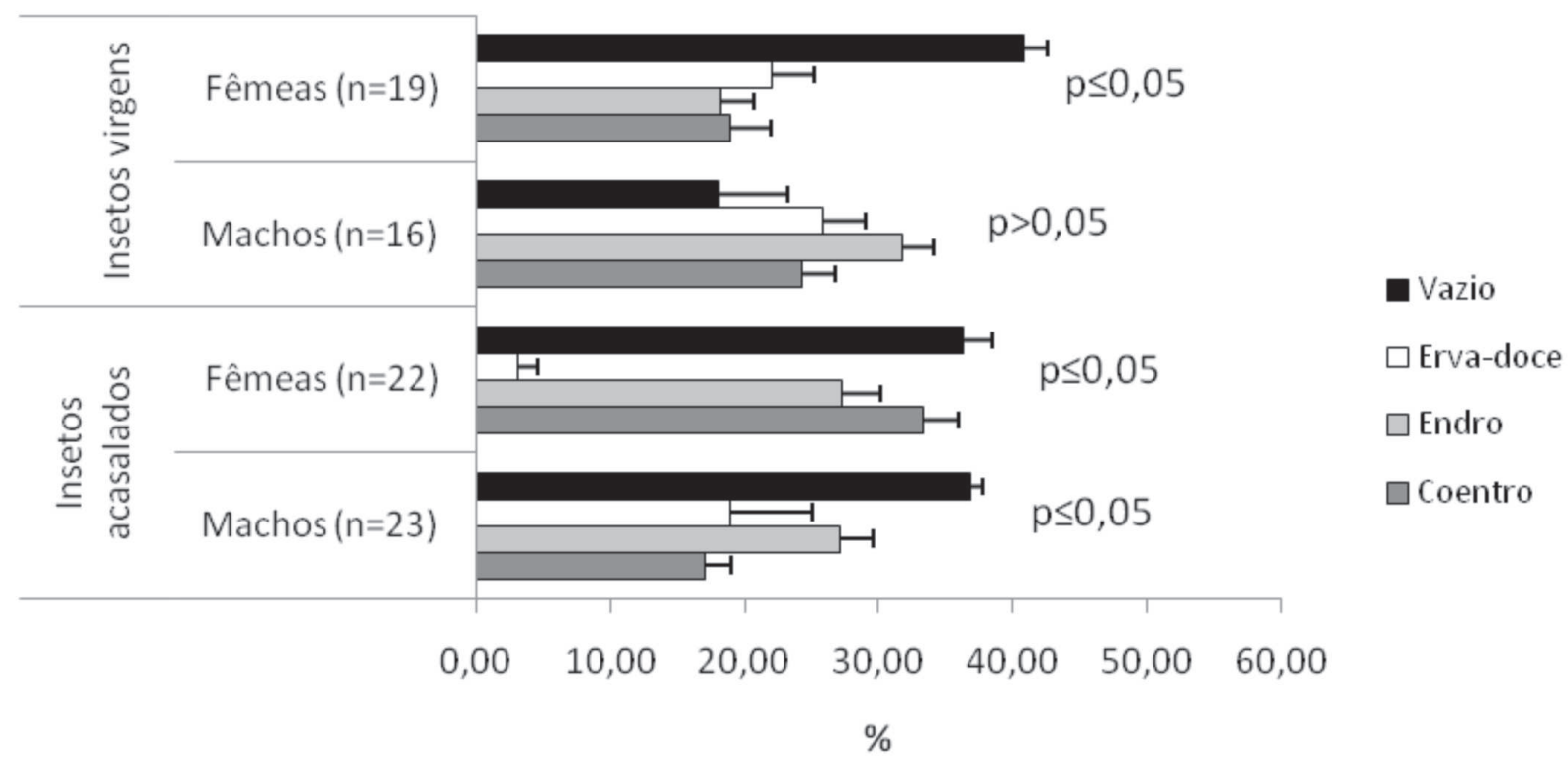

Figura 3. Percentagem de tempo total (médias \pm erro padrão) de machos e fêmeas de Chrysoperla externa, acasalados e virgens, em teste de múltipla escolha em olfatômetro, contendo plantas de coentro, endro e erva-doce com 60 dias após a semeadura. ( $\mathrm{p} \leq 0,05$ representa percentagens não aleatórias pelo teste $\chi^{2}$ ).

que essas espécies podem hospedar uma diversidade de herbívoros que lhes servem de alimento e que podem, não apenas manter, mas, também, atrair crisopídeos e outros inimigos naturais para a área cultivada (Resende et al., 2011). Como exemplo, pode-se citar o pulgão da erva-doce Hiadaphis foeniculi (Passerini, 1860) (Hemiptera: Aphididae), que pode ser utilizado como alimento pelos crisopídeos (Lira \& Batista, 2006).

Quanto ao rendimento de óleo essencial, não houve diferença entre as análises efetuadas com materiais coletados aos 30 e 60 dias, para as três espécies vegetais testadas (Tabela 1).

Os resultados obtidos para o rendimento de óleo essencial em diferentes períodos do desenvolvimento das apiáceas permitem descartar a hipótese de mudanças comportamentais de adultos de C. externa, em função da fenologia das plantas. O estudo desenvolvido por Silva et al. (2003) sobre a relação entre o estádio de desenvolvimento de manjericão (Ocimum basilicum L.) (Lamiaceae) e o teor de óleos essenciais evidenciou aumento da concentração de óleos em plantas mais desenvolvidas, ob-

Tabela 1. Rendimento do óleo (média \pm erro padrão) de coentro, endro e erva-doce extraído aos 30 e 60 dias após a semeadura

\begin{tabular}{lcc}
\hline \multirow{2}{*}{ Plantas } & \multicolumn{2}{c}{ Rendimento do óleo $(\boldsymbol{\%})^{\mathbf{1}}$} \\
\cline { 2 - 3 } & \multicolumn{3}{c}{ dias $^{\text {n.s. }}$} & $\mathbf{6 0}$ dias $^{\text {n.s. }}$ \\
\hline Erva-doce $^{\text {n.s. }}$ & $0,3239 \pm 0,0555^{2}$ & $0,4930 \pm 0,0982$ \\
Endro $^{\text {n.s. }}$ & $0,3221 \pm 0,0902$ & $0,4661 \pm 0,1106$ \\
Coentro $^{\text {n.s. }}$ & $0,1101 \pm 0,0350$ & $0,3062 \pm 0,0691$ \\
\hline
\end{tabular}

${ }^{1}$ Expresso em relação ao peso seco; ${ }^{2}$ Erro padrão calculado a partir

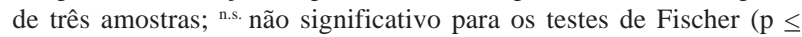
0,05 , na linha) e Tukey ( $\leq \leq 0,05$, na coluna). tendo-se maior produtividade na colheita realizada aos dez meses após o plantio, em relação àquela realizada aos cinco meses. Assim, muitas vezes ocorrem alterações no comportamento dos insetos relacionadas com a preferência pelos voláteis, em função do desenvolvimento fenológico vegetal.

Os compostos de maior concentração no óleo de coentro foram (2E)-decenal e decanal, sendo, ambos os aldeídos, formados a partir da oxidação dos álcoois na planta. Junto a estes também foram compostos majoritári-

Tabela 2. Componentes do óleo essencial de coentro identificados por cromatografia em fase gasosa acoplada a espectrômetro de massas

\begin{tabular}{lccc}
\hline Composto & IRcal & IRlit & \% CG-EM \\
\hline (2E)-decenal & 1260 & 1263 & 20,55 \\
decanal & 1204 & 1201 & 13,77 \\
(2E)-decen-1-ol & 1266 & 1271 & 12,54 \\
decanol & 1270 & 1269 & 11,09 \\
(2E)-dodecenal & 1464 & 1466 & 9,92 \\
(2E)-tridecen-1-al & 1668 & 1568 & 7,92 \\
(2E)-undecanal & 1362 & 1360 & 5,81 \\
undecanal & 1305 & 1306 & 2,67 \\
dodecanal & 1406 & 1408 & 2,59 \\
(2E)-dodecen-1-ol & 1467 & 1471 & 2,52 \\
(2E)-undecenol & 1366 & 1367 & 2,15 \\
undecanol-1 & 1370 & 1370 & 0,79 \\
nonanal & 1103 & 1100 & 0,35 \\
Não identificados & & & 7,33 \\
\hline
\end{tabular}

IRCal = Índice de retenção calculado; IRlit = Índice de retenção obtidos com dados da literatura;

$\%$ CG-EM $=$ Percentagem pela cromatografia gasosa acoplada a espectrômetro de massas. 
os o (2E)-decen-1-ol e decanol (Tabela 2). Análises da composição de óleos essenciais em folhas de coentro, efetuadas por Delaquis et al. (2002), evidenciaram o linalol (25,9\%), (E)-2-decenal $(20,2 \%)$, decanal $(8,4 \%)$ e (E)-2-decen-1-ol (7,9\%) como compostos majoritários e eficazes no controle da bactéria Listeria monocytogenes, por se tratar de álcoois e aldeídos de cadeia longa (6-10 carbonos).

O principal componente do óleo essencial de ervadoce foi o (E)-anetol, com 85,98\% (Tabela 3). Essa concentração representou quase o dobro da encontrada por Tinoco et al. (2007) em folhas desta mesma espécie $(47,8 \%)$. Esse composto também está presente majoritariamente em anis-estrelado, Illicium verum (Hook) (Magnoliaceae), com mais de $90 \%$ da constituição do óleo essencial (Rodrigues et al., 2003; Lima et al., 2008).

O (E)-anetol, ou trans-1-metoxi-4-(prop-1-enil) benzeno, é um composto aromático com característica adocicada, sendo um componente 13 vezes mais doce que o açúcar de mesa, a sacarose. Por causa dessa característica é utilizado na indústria de aromatizantes, principalmente a de licores e balas (Sant'anna et al., 2010). O aroma adocicado exalado pelas plantas de erva-doce, no teste realizado com folhas coletadas aos 30 dias, pode ter sido o fator responsável pela atração dos adultos acasalados, uma vez que estes foram alimentados com dieta à base de mel, nos dias que antecederam o teste.

As naturezas químicas dos compostos encontrados nos óleos essenciais do coentro e da erva-doce foram diferentes. No primeiro, os compostos são alifáticos (hidrocarbonetos de cadeias acíclicas, ou seja, abertas, não apresentando anéis benzênicos em sua composição), enquanto na erva-doce são aromáticos (hidrocarbonetos compostos por um anel benzênico, ou anel aromático).

Tabela 3. Componentes do óleo essencial de erva-doce identificados por cromatografia em fase gasosa acoplada a espectrômetro de massas

\begin{tabular}{lccc}
\hline Composto & IRcal & IRlit & \% CG-EM \\
\hline (E)-anetol & 1285 & 1284 & 85,98 \\
limoneno & 1027 & 1029 & 6,01 \\
metil chavicol & 1195 & 1196 & 2,25 \\
acetato de fenchila & 1229 & 1232 & 1,68 \\
fenchona & 1086 & 1086 & 0,51 \\
á-felandreno & 1004 & 1002 & 0,44 \\
(Z)- $\beta$-ocimeno & 1033 & 1037 & 0,36 \\
Não identificados & & & 2,77 \\
\hline
\end{tabular}

IRCal = Índice de retenção calculado; IRlit = Índice de retenção obtidos com dados da literatura;

$\%$ CG-EM = Percentagem pela cromatografia gasosa acoplada a espectrômetro de massas.
Embora, os voláteis liberados pelo coentro e pela erva-doce possam ter sido atrativos aos adultos de $C$. externa, deve-se salientar que se trata apenas de uma inferência, haja vista não terem sido efetuados testes com os componentes individualizados para comprovação desta hipótese. Desta forma, a partir das observações desta pesquisa, sugerem-se estudos mais detalhados que englobem a composição química dos óleos essenciais destas apiáceas ao longo de todo seu ciclo de vida, objetivando esclarecer os resultados obtidos; bem como testes individuais com as moléculas presentes nesses óleos essenciais, para evidenciar aquelas que são atrativas a adultos de $C$. externa.

\section{CONCLUSÕES}

Aos 30 dias após a semeadura, os adultos virgens de Chrysoperla externa são atraídos por odores de coentro e os adultos acasalados são atraídos por odores de ervadoce.

As plantas de coentro, endro e erva-doce não são atrativas à Chrysoperla externa aos 60 dias após a semeadura.

Não há diferença na quantidade, no rendimento percentual de óleo essencial de coentro, endro e ervadoce, entre si, nem entre os períodos de 30 e de 60 dias após a semeadura.

\section{AGRADECIMENTOS}

Os autores agradecem ao Prof. Luís Cláudio Paterno Silveira e ao Prof. Martín Francisco Pareja, do Departamento de Entomologia, Universidade Federal de Lavras, pelas sugestões e fornecimento de material para realização dos testes com olfatômetro; à Profa. Maria das Graças Cardoso, do Departamento de Química, Universidade Federal de Lavras, pelas análises qualitativas, quantitativas e de rendimento dos óleos essenciais das plantas estudadas neste trabalho; ao Conselho Nacional de Desenvolvimento Científico e Tecnológico (CNPq), à Fundação de Amparo à Pesquisa do Estado de Minas Gerais (FAPEMIG) e à Coordenação de Aperfeiçoamento de Pessoal de Nível Superior (CAPES), pelo auxílio financeiro e concessão de bolsas de estudo.

\section{REFERÊNCIAS}

Adams RP (2007) Identification of essential oil components by gas chromatography/mass spectroscopy. $4^{\text {th }}$ Edition. Allured Publ. Corp, Carol Stream. 804p.

Andrade MA, Cardoso MG, Batista LR, Mallet ACT \& Machado SMF (2012) Óleos essenciais de Cymbopogon nardus, Cinnamomum zeylanicum e Zingiber officinale: composição, atividades antioxidante e antibacteriana. Revista Ciência Agronômica, 43:399-408. 
Carvalho CF \& Souza B (2002) Potencial de insetos predadores no controle biológico aplicado. In: Parra JRP, Botelho PSM, CorrêaFerreira BS \& Bento JMS. Controle biológico no Brasil: Parasitóides e Predadores. São Paulo, Manole. p.191-208.

Costa RIF, Carvalho CF, Souza B \& Loreti J (2003) Influência da densidade de indivíduos na criação de Chrysoperla externa (Hagen, 1861) (Neuroptera Chrysopidae). Ciência e Agrotecnologia, 27:1539-1545.

Delaquis PJ, Stanich K, Girard B \& Mazza G (2002) Antimicrobial activity of individual and mixed fractions of dill, cilantro, coriander and eucalyptus essential oils. International Journal of Food Microbiology, 74:101-109.

Freitas S. (2002) O uso de crisopídeos no controle biológico de pragas. In: Parra JRP, Botelho PSM, Corrêa-Ferreira BS \& Bento JMS. Controle Biológico no Brasil: Parasitóides e Predadores. São Paulo, Manole. p.209-224

Kessler A \& Baldwin IT (2001) Defensive function of herbivore-induced plant volatile emissions in nature. Science, 291:2141-2144.

Lima RK, Cardoso MG, Morais JC, Vieira SS, Melo BA \& Filgueiras CC (2008) Composição dos óleos essenciais de Anis-estrelado Illicium verum L. e de Capim-limão Cymbopogon citratus (DC.) Stapf: Avaliação do efeito repelente sobre Brevicoryne brassicae (L.) (Hemiptera: Aphididae). BioAssay, 3:1-6

Lira RS \& Batista JL (2006) Aspectos biológicos de Chrysoperla exter$n a$ alimentados com pulgões da erva-doce. Revista de Biologia e Ciência da Terra, 6:20-35.

Lixa AT, Campos JM, Resende ALS, Silva JC, Almeida MMTB \& AguiarMenezes EL (2010) Diversidade de Coccinellidae (Coleoptera) em plantas aromáticas (Apiaceae) como sítios de sobrevivência e reprodução em sistema agroecológico. Neotropical Entomology, 39:354359 .

Medeiros MA, Sujii ER \& Morais HC (2009) Effect of plant diversification on abundance of South American tomato pinworm and predators in two cropping systems. Horticultura Brasileira, 27:300-306.

Reddy GVP, Holopainen JK \& Guerrero A (2002) Olfactory responses of Plutella xylostella natural enemies to host pheromone, larval frass, and green leaf cabbage volatiles. Journal of Chemical Ecology, 28:131143.

Resende ALS, Viana AJS, Oliveira RJ, Aguiar-Menezes EL, Ribeiro RLD, Ricci MSF \& Guerra JGM (2010) Consórcio couve-coentro em cultivo orgânico e sua influência nas populações de joaninhas. Horticultura Brasileira, 28:41-46.

Resende ALS, Lixa AT, Santos CMA, Souza SAS, Guerra JGM \& AguiarMenezes EL (2011) Comunidade de Joaninhas (Coleoptera: Coccinellidae) em Consórcio de Couve (Brassica oleraceae var. acephala) com Coentro (Coriandrum sativum) sob Manejo Orgânico. Revista Brasileira de Agroecologia, 6:81-89.
Rodrigues VN, Rosa PTV, Marques MOM, Petenate AJ \& Meirele MAA (2003) Supercritical Extraction of essential oil from aniseed (Pimpinella anisum L.) using $\mathrm{CO}_{2}$ : solubility, kinetics, and composition data. Jounal of Agricultural and Food Chemistry, 51:15181523 .

Rosado LDS, Pinto JEBP, Botrel PP, Bertolucci SKV, Niculau ES \& Alves PB (2011) Influência do processamento da folha e tipo de secagem no teor e composição química do óleo essencial de manjericão cv. Maria Bonita. Ciência e Agrotecnologia, 35:291-296.

Sant'anna HLS, Santos OSN, Santos CRS, Martins CY, Santos MB, Almeida MA, Silva F, Martins GN \& Ledo CAS (2010) Longevidade pós-colheita de alpínia [Alpinia purpurata (Vieill.) K. Schum.] tratada com soluções de sacarose e extratos aquosos naturais. Revista Brasileira de Plantas Medicinais, 12:269-277.

Silva F, Santos RHS, Diniz ER, Barbosa LCA, Casali VWD \& Lima RR (2003) Teor e composição do óleo essencial de manjericão (Ocimum basilicum L.) em dois horários e duas épocas de colheita. Revista Brasileira de Plantas Medicinais, 6:33-38.

Simões CMO \& Spitzer V (2000) Óleos voláteis. In: Simões CMO, Schenkel EP, Gosmann G, Mello JCP, Mentz LA \& Petrovick PR. Farmacognosia: da planta ao medicamento. Porto Alegre, Universidade Federal do Rio Grande do Sul. p.394-412.

Tinoco MT, Martins MR \& Cruz-Morais J (2007) Atividade antimicrobiana do óleo essencial do Foeniculum vulgare Miller. Revista de Ciências Agrárias, 30:448-454.

Togni PHB, Frizzas MR, Medeiros MA, Nakasu EYT, Pires CSS \& Sujii ER (2009) Dinâmica populacional de Bemisia tabaci biótipo B em tomate monocultivo e consorciado com coentro sob cultivo orgânico e convencional. Horticultura Brasileira, 27:183188 .

Togni PHB, Laumann RA, Medeiros MA \& Sujii ER (2010) Odour masking of tomato volatiles by coriander volatiles in host plant selection of Bemisia tabaci biotype B. Entomologia Experimentalis et Applicata, 136:164-173.

Van Den Dool H \& Kratz DJ (1963) A generalization of the retention index system incluing linear temperature programmed gas-liquid partition chromatography. Journal of Chromatography, 11:463-467.

Zhu J, Obrycki JJ, Ochieng SA, Baker TC, Pickett JA \& Smiley D (2005) Attraction of two lacewing species to volatiles produced by host plants and aphid prey. Naturwissenschaften, 92:277281. 